\title{
NF-KB: pivotal mediator or innocent bystander in atherogenesis?
}

\author{
Tucker Collins ${ }^{1}$ and Myron I. Cybulsky ${ }^{2}$ \\ ${ }^{1}$ Department of Pathology, Brigham and Women's Hospital and Harvard Medical School, Boston, Massachusetts, USA \\ ${ }^{2}$ Department of Laboratory Medicine and Pathobiology, University of Toronto, Toronto General Research Institute, Toronto, \\ Ontario, Canada \\ Address correspondence to: Tucker Collins, Department of Pathology, Brigham and Women's Hospital, 221 Longwood Avenue, Boston, \\ Massachusetts 02115, USA. Phone: (617) 732-5990; Fax: (617) 278-6990; E-mail: tcollins@rics.bwh.harvard.edu.
}

Atherosclerosis and its clinical manifestations of heart attack, stroke, and peripheral vascular insufficiency are a major cause of morbidity and mortality among both men and women. Multiple risk factors including hypertension, diabetes mellitus, smoking, and lipoprotein disorders are involved in the pathogenesis of this chronic inflammatory disease of arteries.

The development of early atherosclerotic lesions can be subdivided into initiation (formation of small fatty streaks), expansion (vertical and lateral growth and coalescence of fatty streaks), and progression to plaques (intimal smooth muscle cell recruitment, collagen deposition, and formation of a fibrous cap) (reviewed in ref. 1). During the initiation and expansion of fatty streaks, circulating monocytes are recruited to the arterial intima where they are transformed into lipid-engorged macrophage foam cells. The arterial endothelium in these regions is activated and expresses inducible leukocyte adhesion molecules and chemokines. Production of cytokines and growth factors within lesions may amplify monocyte recruitment, stimulate macrophage proliferation, and induce migration of smooth muscle cells from the media to the intima of the vessel. Intimal smooth muscle cells deposit collagen and other ECM proteins, leading to the formation of a fibrous cap. Although clinically significant complications of atherosclerosis, such as plaque ulceration, rupture, and thrombosis, occur in established or advanced atherosclerotic plaques, understanding the mechanisms of early lesion formation offers the hope of intervening to delay or prevent lesion progression and complications. A select set of transcription factors may be critical in both the initiation and expansion of lesions, as well as in protecting the vessel wall from the formation of atherosclerotic lesions. In this overview, we will focus on one transcription factor, NF- $\mathrm{KB}$, whose activation has been linked to the onset of atherosclerosis.

NF- $\kappa B$ is composed of members of the Rel family that share a 300 amino acid region, known as the Rel homology domain, which mediates dimerization, nuclear translocation, DNA binding, and interaction with NF- $\kappa B$ inhibitors (reviewed in ref. 2). Activation of NF- $\mathrm{KB}$ is controlled by a family of inhibitors, or I $\kappa B s$, that bind to NF- $\kappa B$ dimers and mask the nuclear localization sequence of NF- $\mathrm{KB}$, thus retaining the entire complex in the cytoplasm. Diverse stimuli activate NF- $\kappa \mathrm{B}$, through the phosphorylation and activation of the IKB kinase (IKK) complex. This complex consists of IKK- $\alpha$ and IKK- $\beta$ heterodimers, a number of IKK- $\gamma$ subunits, and possibly other components that have less certain significance. The activated IKK complex specifically phosphorylates the IKBs, which are then rapidly polyubiquitinated, targeting them for degradation by the proteosome. Following release from the inhibitor, NF- $\kappa \mathrm{B}$ dimers translocate from the cytoplasm to the nucleus, where they bind target genes and stimulate transcription (Figure 1). NF- $\kappa$ B activates a variety of target genes relevant to the pathophysiology of the vessel wall, including cytokines, chemokines, and leukocyte adhesion molecules, as well as genes that regulate cell proliferation and mediate cell survival. NF- $\kappa B$ also activates the $I \kappa B \alpha$ gene, thus replenishing the cytoplasmic pool of its own inhibitor. Restored

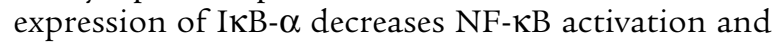
diminishes expression of NF- $\kappa \mathrm{B}-$ dependent genes. The NF- $\kappa \mathrm{B} / \mathrm{I} \kappa \mathrm{B}-\alpha$ autoregulatory system ensures that the induction of NF- $\kappa B$ is transient and that the activated cell returns to a quiescent state. Physiological modulation and pathological activation of the NF- $\mathrm{\kappa B}$ system may contribute to the changes in gene expression that occur during atherogenesis.

\section{Activated NF-KB and its target gene products in atherosclerotic lesions}

Activated NF- $\kappa \mathrm{B}$ has been identified in situ in human atherosclerotic plaques but is absent or nearly so in vessels devoid of atherosclerosis. Using a $\mathrm{mAb}$ that recognizes the IKB binding region on the p 65 component of the transcription factor, Brand et al. (3) detected activated NF- $\mathrm{\kappa B}$ in the intima and media of atheromatous areas of atherosclerotic lesions in smooth muscle cells, macrophages, and endothelial cells. Little activated NF- $\mathrm{KB}$ is detected in healthy vessels. In situ analysis of p50 and p65 in the normal vessel wall reveals diffuse cytoplasmic expression. No nuclear accumulation is seen with either the p50 or p65 antisera. Thus, in medial smooth muscle cells of the normal vessel wall, NF- $\kappa B$ proteins are restricted to the cytosol, suggesting that the regulatory system is quiescent. However, within human atheromas, the nuclei 


\section{Figure 1}

Schematic representation of NF- $\kappa B$ as an integrator in atherogenesis. Many of the diverse agents associated with the onset of lesion formation interact with specific receptors. Angiotensin II (Ang II), cytokines, advanced glycosylation end products (AGEs), or oxidized lipids bind to the Ang II receptor (AT1), cytokine receptors, the receptor for AGEs (RAGE), or the scavenger receptor (SR), respectively. LPS interacts with a complex of LPS-binding protein (LBP), CD14 and a toll-like receptor (TLR). The mechanisms by which hemodynamic forces are perceived by vascular cells are only partially appreciated, but may involve specific receptors or links between the cytoskeleton, integrins, and ECM. Ligand binding by most of these receptors results in recruitment of adaptor proteins and activation of intermediate kinases (not shown). These events ultimately lead to the activation of an IKK complex. The best characterized kinase complex consists of IKK- $\alpha$, IKK- $\beta$, and the structural component IKK- $\gamma$, although other similar complexes may exist. The activated IKK complex specifically phosphorylates the $I K B s$, which then undergo a rapid polyubiquitination process prior to degradation by the proteosome. Following release from the inhibitor, NF- $\mathrm{KB}$ dimers translocate from the cytoplasm to the nucleus, where they bind target genes and stimulate transcription of specific sets of genes relevant to the pathophysiology of the vessel wall.

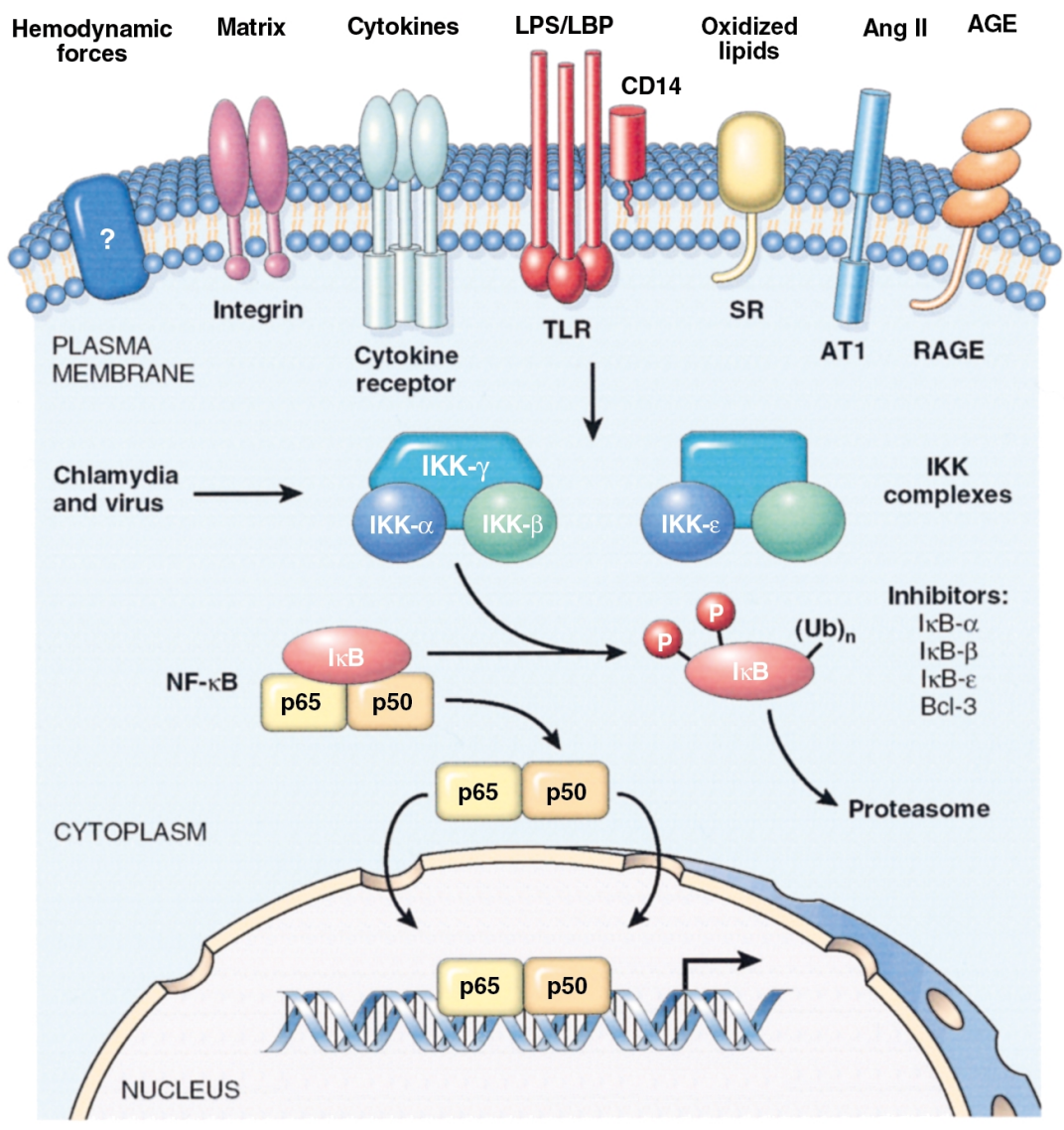

of intimal smooth muscle cells contain both Rel proteins, suggesting that the transcription factor is activated. More recently, activated NF- $\mathrm{\kappa B}$ was detected in intimal cells found in coronary arteries of pigs placed on a hypercholesterolemic diet (4). In models of arterial injury, NF- $\mathrm{KB}$ is activated in both endothelial and smooth muscle cells. Collectively, these observations support a role for NF-KB in atherosclerosis.

Multiple genes whose products are putatively involved in the atherosclerotic process are regulated by NF-KB. Leukocyte adhesion molecules, such as VCAM-1, ICAM-1, and E-selectin, as well as the chemokines (chemoattractant cytokines) monocyte chemoattractant protein-1 (MCP-1) and IL-8, help recruit circulating mononuclear leukocytes to the arterial intima. The induction of other NF- $\mathrm{kB}$-dependent genes such as tissue factor could tip the pro/anti-coagulant balance on the endothelial cell surface towards coagulation. Still other products of target genes, including cyclin D1, may induce cell proliferation or stimulate cell survival at the sites of lesion formation (see below). Collectively, the coordinate induction of NF-KB-dependent genes may exert a substantial atherogenic effect on the vessel wall. If the response persists, it may stimulate migration and proliferation of smooth muscle cells, as well as ongoing recruitment of circulating monocytes. Activation of these cells, in turn, can lead to the release of additional cytokines and chemokines, as well as the production of growth factors. Therefore, NF- $\mathrm{KB}$ may be key both to initial responses to the atherogenic signal and to subsequent amplification steps during the expansion and progression of lesions.

The induction of VCAM-1 at sites of atherosclerotic lesion formation is an example of inducible NF$\mathrm{\kappa B}$-dependent gene expression during formation of early atherosclerotic lesions. VCAM-1 is an adhesion molecule whose expression is induced by inflammatory cytokines on endothelial, smooth muscle, and some nonvascular cells. Its expression has been studied extensively in animal models of atherogenesis and in human tissues. These studies provide insights into potential pathophysiological functions of this gene as well as NF- $\mathrm{KB}$ activation, since the promoter of $V C A M 1$ contains two consensus NF- $\kappa B$ sites that are required for cytokine-induced expression. In normal mouse and rabbit aortas, low levels of VCAM-1 are found in endothelium only at sites predisposed to atherosclerotic lesion formation (5).

Hypercholesterolemia, whether in apoE knockout $\left(\right.$ apo $\left.E^{-/}\right)$mice or cholesterol-fed rabbits, upregulates 
endothelial VCAM-1 expression in these regions. In rabbits, introducing cholesterol into the diet results in readily detectable VCAM-1 expression in the ascending aorta at 1 week, which precedes intimal macrophage foam cell lesion formation. Once atherosclerotic lesions are established, VCAM-1 remains largely restricted to lesions, although its expression shifts from endothelial cells - where it predominates in early lesions - to the intimal cells as lesions progress (5). Expression in endothelium is most intense at lesion borders and immediately adjacent to lesions, sites of abundant mononuclear leukocyte adherence; this pattern is found in dietary and heritable (Watanabe) hyperlipidemic rabbits (6) and LDL receptor knockout ( $\left.L D L-R^{-/-}\right)$mice (5). VCAM-1 expression can be detected in advanced human atherosclerotic lesions, although it is somewhat variable and found mostly in intimal cells and neovessels.

Once leukocytes are adherent at sites of developing atherosclerotic lesions, they enter the wall directed by NF-KB-dependent chemokines, such as MCP-1. This chemoattractant chemokine is highly expressed in human atherosclerotic lesions and in animal models of atherogenesis, where it is thought to contribute to the initiation and the amplification of monocyte recruitment to the artery wall. The expression of both VCAM-1 and MCP-1 at sites of atherosclerotic lesion formation is an excellent example of how coordinate induction of NF- $\mathrm{kB}$-dependent genes may be necessary for efficient formation of early atherosclerotic lesions. Mononuclear leukocyte recruitment from blood into tissues requires more than just upregulation of VCAM-1 expression on endothelium. The primary ligand of VCAM-1, the $\alpha 4 \beta 1$ integrin, which is expressed constitutively on all leukocyte types except mature neutrophils, has a low ligand-binding activity, as do most integrins on circulating leukocytes. Only following stimulation by chemokines, such as MCP-1, at the site of inflammation will leukocyte integrin ligand-binding function increase and mediate strong adhesion via VCAM-1.

The complex roles of various genes in atherogenesis can be studied in murine models using transgenic approaches. Mouse models of atherosclerosis include $a p o E^{-1-}, L D L R^{-1-}$, and human $a p o B$ transgenic mice, which develop lesions throughout the arterial tree. Their lesion distribution pattern and morphologic features share many similarities with human atherosclerosis, suggesting that similar pathogenic mechanisms may be involved. The standard approach is to breed mice deficient in a candidate gene into the $a p o E^{-/-}$or $L D L R^{-/-}$ background and evaluate the extent of lesion formation in the aortic root or throughout the aorta. Such studies show that both MCP-1 and the adhesion molecules are crucial in atherogenesis. To demonstrate that MCP-1 and its leukocyte receptor, the $\mathrm{CC}$ chemokine receptor CCR2, play key roles in atherogenesis, Boring et al. (7) crossed CCR2-deficient animals with mice lacking apoE. The resulting CCR2/apoE double-null mice exhibited a reduction in mean aortic lesion area, despite unchanged serum lipid levels. Similarly, MCP-1/LDLR double-knockout mice enjoy dramatic protection from atherosclerotic lesion formation, compared with LDLRdeficient control mice (8). These initial studies provided compelling evidence that MCP-1 and its receptor play a critical role in the initiation of atherosclerosis. Deficiencies of various leukocyte adhesion molecules, or combinations of adhesion molecules, also result in reduced atherosclerotic lesion formation in murine models. Although deficiency of VCAM-1 results in embryonic lethality, recent studies using mice that express markedly reduced levels of VCAM-1 reveal a substantial reduction in atherosclerotic lesions (M.I. Cybulsky, unpublished observations).

\section{$\mathrm{NF}-\mathrm{KB}$ activation in atherogenesis}

Oxidative stress. The various risk factors for atherosclerosis, including hyperlipidemia, hypertension, and diabetes, have in common the generation of intracellular oxidative stress. In respiring cells, small amounts of oxygen are reduced to reactive oxygen species. These reactive oxygen intermediates, produced in mitochondria, peroxisomes, and the cytosol, are scavenged by cellular defending systems, including enzymatic and nonenzymatic antioxidants. A state of moderately increased levels of intracellular reactive oxygen species is referred to as oxidative stress. Cells respond to this stress by increasing the levels of antioxidants and altering the intracellular reduction-oxidation (redox) state.

$\mathrm{NF}-\mathrm{\kappa B}$ is one of the transcription factors that may be controlled by the redox status of the cell (reviewed in ref. 9). Indeed, generation of reactive oxygen species may be a common step in all of the signaling pathways that lead

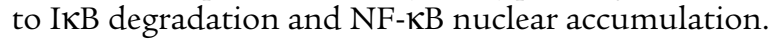
Support for this concept comes from a variety of studies showing that the diverse agents that can activate NF- $K B$ also elevate levels of reactive oxygen species and that chemically distinct antioxidants, as well as overexpression of antioxidant enzymes, can inhibit NF- $\kappa \mathrm{B}$ activation. However, a direct role of reactive oxygen species in signaling to NF- $\mathrm{KB}$ remains to be proven. It is unlikely that either NF- $\kappa B$ or I $\kappa B$ is directly activated by oxidation. Most of the evidence suggests that oxidants induce, and antioxidants diminish, some aspect of the signaling events leading to the phosphorylation, ubiquitination, and degradation of IкB. Understanding how redox processes influence the NF- $\kappa B$ system is an important unresolved issue that may provide novel mechanistic insights into vascular dysfunction.

Dyslipidemia. As with oxidative stress, the presence of elevated and modified lipoproteins is strikingly associated with atherogenesis. The accumulation of LDL in the arterial intima is an early event in lesion formation and continues as lesions advance. When LDL particles 
become trapped in an artery, they undergo a progressive oxidation in a localized microenvironment that is relatively sequestered from plasma antioxidants. The nature of the cellular signals that lead to the generation of the oxidized lipids is not resolved, but once oxidized, the LDL particles can be internalized by macrophages by means of the scavenger receptors on the surfaces of these cells. Removal of oxidized LDL is an important part of the protective function of macrophages, because it minimizes the effects of modified LDL on endothelial cells and smooth muscle cells. The internalization of oxidized LDL leads to the accumulation of lipid peroxides and facilitates the accumulation of cholesterol esters, resulting in the formation of foam cells.

Several studies suggest that elevated levels of native LDL, as well as oxidized LDL, act as pro-oxidant signals regulating vascular cell gene expression. Shortterm exposure of monocytes to oxidized LDL activates NF- $\mathrm{KB}$ and induces the expression of target genes, although longer exposure may suppress these responses (10). Nonmodified (native) LDL and minimally oxidized LDL stimulate endothelial cells to produce a series of NF- $\kappa B$-dependent chemokines and adhesion molecules (11). Similarly, components of oxidized LDL such as lysophosphatidylcholine induce expression of mononuclear leukocyte adhesion molecules and can activate NF-KB in endothelial cells (12). Production of these chemokines may help expand the inflammatory response by stimulating proliferation of resident macrophages and the recruitment of new monocytes into lesions. Additionally, the inflammatory cytokines present in lesions can increase binding of LDL to endothelium and smooth muscle cells and increase the expression of the LDL receptor, leading to further inflammation.

To study LDL oxidation in vivo, Calara et al. (13) injected human LDL particles into a rat model and observed that these lipoproteins localized in the arterial wall, where they underwent oxidative modification accompanied by an activation of the endothelial NF- $\kappa B$ system and expression of NF-kB-dependent genes (13). Similar studies show that VLDL causes arterial activation of NF- $\mathrm{KB}$ and increased expression of NF$\kappa \mathrm{B}$-dependent genes (14). Collectively, these studies suggest that both LDL and VLDL may promote atherosclerosis in vivo in vascular cells, at least in part, by activating NF- $\mathrm{KB}$ in vascular cells.

Oxidized lipids in the vessel wall may regulate vascular cell gene expression by activating PPARs. These receptors are ligand-activated transcription factors belonging to the nuclear receptor family. They function as regulators of lipid and glucose metabolism and affect both cellular differentiation and apoptosis. PPAR $\alpha$ stimulates $\beta$ oxidation of fatty acids, while PPAR $\gamma$ elicits adipocyte differentiation and promotes lipid storage. Fatty acids and eicosanoids are natural PPAR ligands, while the hypolipidemic fibrates and the antidiabetic glitasones are synthetic ligands for PPAR $\alpha$ and PPAR $\gamma$, respectively. Components of oxidized LDL may activate PPAR $\gamma$ and regulate macrophage gene expression in ways that lead to foam-cell formation (15). However, several recent findings support the concept that the PPARs also suppress chronic inflammation in the vessel wall. Thus, PPAR $\alpha$-deficient mice exhibit a prolonged response to inflammatory stimuli, and PPAR activators inhibit the expression of a number of proteins (such as IL-2, IL-6, IL-8, iNOS, TNF- $\alpha$ and matrix metalloproteinases) involved in the inflammatory response in vascular cells. Finally, PPAR $\gamma$-specific agonists inhibit the development of atherosclerosis in LDL receptor-deficient male mice (16). Interestingly, PPARs inhibit the vascular inflammatory response, at least in part, by interfering with multiple steps in the NF- $\mathrm{\kappa B}$ signaling pathway.

Hypertension and angiotensin II. Hypertension is an established risk factor for atherosclerosis. Patients with hypertension frequently have elevated levels of angiotensin II (Ang II), the principal product of the renin-angiotensin system. Ang II is a potent vasoconstrictor that may be related to atherosclerosis because of its effect on blood pressure or its effects on smooth muscle cell growth. Additionally, Ang II elicits an inflammatory response in both endothelial cells and vascular smooth muscle cells that appears to be dependent upon the generation of oxidant stress and NF- $\kappa B$ activation. Ang II mediates both the hemodynamic and the inflammatory effects through the Ang II receptor (AT1) (Figure 1). In vascular cells, Ang II generates oxidant stress by the induction of superoxide via NADH oxidase, or by stimulating the generation of reactive oxygen species in the mitochondria. Ang II-induced oxidative stress is associated with NF- $\mathrm{KB}$ activation and VCAM-1 induction in endothelial cells (17) and with NF-KB-dependent transcription of IL-6 in vascular smooth muscle cells (18). In the rat vasculature, administration of Ang II activates NF- $\mathrm{KB}$ and induces the expression of both IL- 6 and VCAM-1 (19). Selective inhibitors of AT1, as well as inhibitors of IKB proteolysis, block these responses. Inflammatory activation of the vessel wall by a dysregulated angiotensin system, potentially mediated by NF- $\kappa \mathrm{B}$, may contribute to the pathogenesis of atherosclerosis.

Diabetes and the effects of advanced glycation end products and the receptor for advanced glycation end products. Diabetes is another major risk factor for atherosclerosis. Diabetes-associated hyperglycemia produces intracellular oxidant stress that can lead to vascular dysfunction. Formation of advanced glycation end products (AGEs) due to elevated nonenzymatic glycation of proteins is accompanied by oxidative reactions that generate oxygen free radicals under hyperglycemic conditions. Once produced, AGEs can alter cellular function by binding to the receptor for AGEs (RAGE) (reviewed in ref. 20). Binding of AGEs to RAGE results in intracellular oxi- 
dant stress and activation of NF- $\mathrm{KB}$ in cultured endothelial cells. Hyperglycemia activates NF- $\kappa B$ and promotes leukocyte adhesion to the endothelium through upregulation of cell surface expression of VCAM-1 and other adhesion proteins (21). Similarly, hyperglycemia induces activation of NF- $\mathrm{KB}$ in vascular smooth muscle cells. This may contribute to shifting the tone of the arterial wall, as well as increasing smooth muscle cell proliferation, leading to increased intimal wall thickness. Thus, NF- $\mathrm{KB}$ activation is an early event in response to increases in glucose, and may elicit multiple pathways contributing to vascular complications.

Homocysteine and NF- $\kappa B$ activation. Increased plasma levels of homocysteine metabolism are an independent risk factor for atherosclerosis and thromboembolic disease. It is postulated that homocysteine induces endothelial dysfunction, leading to a prothrombotic environment (reviewed in ref. 22). Although the mechanisms by which this occurs are uncertain, attempts have been made to establish in vitro model systems to evaluate the effects of homocysteine on cultured cells. This approach suggests that homocysteine creates oxidative stress by altering the redox thiol status of the cell (23). A consequence of these effects is the activation of NF-KB, possibly by a homocysteine-generated reactive oxygen species. Exposure of cultured vascular smooth muscle cells to physiologic levels of homocysteine leads to a marked increase in vascular smooth muscle proliferation in vitro, an effect that is due in part to increased expression of cyclin D1 (22). Activation of $\mathrm{NF}-\kappa \mathrm{B}$ may contribute to the mitogenic effects of homocysteine, since NF- $\kappa B$ activates cyclin D1 expression (24) and NF- $\mathrm{KB}$ activity is important for proliferation of vascular smooth muscle cells. Additionally, homocysteine has proinflammatory effects on both endothelial and smooth muscle cells. Homocysteine increases leukocyte rolling, adherence, and transmigration in mesenteric venules by upregulation of adhesion molecules and suppression of endothelial-derived nitric oxide (NO) (25). This suggests that homocysteine inhibits the important role of endothelial NO in preventing endothelial dysfunction. In cultured aortic smooth muscle cells, homocysteine leads to both an increase in NO production and a NF- $\mathrm{KB}$-mediated increase in the expression of inducible NO synthase (iNOS) (22). Upregulation of iNOS in smooth muscle cells may generate oxidative stress and contribute to the inflammatory response that characterizes the atherogenesis associated with hyperhomocysteinemia.

Infectious agents. Atherosclerosis is considered to be a chronic inflammatory disease of the arterial wall. There is increasing interest in the possible role of microbial agents as stimuli that trigger the arterial inflammation. There is a correlation between atherosclerosis and the presence of at least two classes of microorganisms: herpesviruses, such as cytomegalovirus (CMV), and Chlamydia pneumonia (reviewed in ref. 26). Briefly, there is seroepidemiologic evidence that supports the involvement of both types of infectious agents in atherosclerosis, and preliminary findings that treatment with antibiotics might reduce recurrent coronary events. In studies localizing these infectious agents to human arterial lesions, both types of organisms are identified in atheromas in coronary arteries, as well as in other vessels. Additionally, both pathogens can produce lesions in experimental animals and can infect vascular cells, generating products that cause both endothelial and smooth muscle cells to become dysfunctional. In endothelial cells these changes result in increased expression of leukocyte adhesion molecules, cytokines, and procoagulants; smooth muscle cells are stimulated to proliferate and produce cytokines.

CMV infection is associated with atherosclerosis, as well as restenosis and allograft vasculopathy. In vascular smooth muscle cells, CMV infection generates intracellular reactive oxygen intermediates and activates NF- $\mathrm{KB}$ (ref. 27; see also Hiscott et al., this Perspective series, ref. 28). These initial signaling events may be necessary for expression of viral components and viral replication. Additionally, virally induced NF- $\mathrm{KB}$ may increase the expression of cellular genes, such as cytokines and the adhesion molecule ICAM-1, that are involved in the inflammatory response. In endothelial cells, CMV infection is associated with a modulation of adhesion molecule expression (29). Additionally, herpesviruses can act as prothrombotic agents by activating the coagulation cascade (reviewed in ref. 30). These virally mediated changes may exacerbate the host inflammatory response associated with atherosclerotic lesion formation.

There is provocative evidence for Chlamydia as a trigger of arterial inflammation. The chlamydial heat shock protein 60 (HSP 60) is found in human athero$\mathrm{ma}(31)$. It activates NF- $\mathrm{KB}$ and induces the expression of a series of leukocyte adhesion molecules in endothelial cells and stimulates the production of TNF- $\alpha$ and matrix metalloproteinase expression in macrophages. Thus, consistent with the hypothesis that NF- KB plays a key integration role in atherosclerosis, some microbial products activate NF- $\mathrm{KB}$ and initiate a series of dysfunctional changes in vascular cells that can contribute to the pathophysiology of the atheroma.

Atheroprotection and suppression of the NF-KB system

Because of its exposure to blood components, the endothelium is susceptible to oxidative stress produced by diet-derived components. Dietary fats, rich in unsaturated fatty acids, become atherogenic by increasing oxidative stress in the vessel wall. Activation of stress responsive transcription factors like NF- $\mathrm{KB}$ would promote the critical cellular events during early atherosclerosis.

Certain nutrients have in common the ability to suppress the NF- $\mathrm{KB}$ system and diminish endothelial dysfunction. Dietary long-chain unsaturated fatty acids 
are associated with diminished risk of atherosclerosis. Fatty acids of the omega 3 family, such as n-3 fatty acid docosahexaenoate (DHA), inhibit cytokine-stimulated expression of endothelial-leukocyte adhesion molecules and soluble cytokine production in the range of nutritionally achievable plasma concentrations. In contrast, saturated fatty acids did not inhibit cytokineinduced endothelial activation. DHA reduces production of hydrogen peroxide and inhibits NF- $\mathrm{KB}$ activation (32). Similarly, oleic acid, the main component in olive oil, has been implicated in the antiatherogenic effects of a Mediterranean diet. Oleic acid inhibits NF- $\mathrm{KB}$ activation and cytokine-induced expression of adhesion molecules. Although less potent than polyunsaturated fatty acids in inhibiting endothelial activation, oleic acid may contribute to the prevention of atherogenesis, at least in part, by inhibiting NF- $\mathrm{KB}$ and diminishing the expression of genes involved in monocyte recruitment in the arterial intima.

Atherogenesis is also diminished by a variety of other agents that reduce oxidant stress. Human studies link antioxidants with a reduction in cardiovascular disease (reviewed in ref. 33). Strategies for defense against oxidative stress and the resulting damage that it generates include the production of antioxidant enzymes, including catalase, several forms of superoxide dismutase, and glutathione peroxidase. These antioxidant enzymes are complemented by small-molecule antioxidants, some of which are derived from the diet as vitamins. Antioxidant nutrients such as vitamins $\mathrm{A}, \mathrm{C}$, and $\mathrm{E}$, as well as $\beta$-carotene, zinc, selenium, and magnesium, may inhibit early atherogenesis by inhibiting LDL oxidation or by decreasing cellular production and release of reactive oxygen species in endothelial cells. Incorporation of lipid-soluble antioxidants into LDL protects it against oxidation and leads to the reduced formation of oxidized LDL. In addition, incorporation of antioxidants into vascular cells may reduce vascular cell oxidative stress. Some of these biologically relevant antioxidants apparently inhibit NF- $\kappa \mathrm{B}$ activation in macrophages and cultured endothelial cells (34) and decrease oxidative stress in the porcine coronary artery (35). By diminishing NF- $\mathrm{KB}$ activation, the antioxidant vitamins would, at least in part, diminish the cellular responses to oxidized LDL, reducing monocyte adhesion, foamcell formation, and cytotoxicity to vascular cells, and improving vascular function. Taken together these findings are consistent with the concept that agents that diminish oxidant stress may stabilize the NF- $\mathrm{KB}$ system and decrease atherosclerotic lesion formation.

\section{Activation of NF-kB by proatherogenic agents}

Despite the numerous correlations between atherogenesis and the activity of the NF- $\kappa B$ system, very little is known about how the different signals associated with atherogenesis activate or suppress the transcription factor. If the well characterized IKK complex integrates the diverse proatherogenic signals, it will be important to determine at what point the activation pathways converge. These proatherogenic signaling systems may link with the IKK complex by binding to established components of the complex, such as IKK- $\gamma$, or perhaps by recruiting novel adapters. These proteins could act as scaffolds that tether the primary sensing mechanism to the IKK complex; they could direct the assembly of IKK complexes in response to signaling; or the adapters could control the deactivation and disassembly of the complex after stimulation. Alternatively, the proatherogenic signaling systems may intersect with one of the intermediate kinases, such as the NF- $\mathrm{KB}$-inducing kinase (NIK), which would, in turn, activate the IKK complex.

Another general possibility is that these proatherogenic signaling systems intersect with a novel kinase complex that is responsive to some of the proatherogenic signals. Recently a new inducible kinase complex was identified that is distinct from the well characterized IKK complex (36). This novel complex contains another IKK family member, designated IKK- $\varepsilon$, and one or more associated kinases that can phosphorylate both critical serine residues of IKB- $\alpha$ in response to phorbol 12-myristate 13-acetate (PMA). Proatherogenic signaling systems may activate NF- $\kappa B$ through this new kinase complex, or possibly through multiple kinase complexes.

The signals generated by proatherogenic stimuli could activate kinases that are unrelated to the known IKK complexes. Phosphorylation of the amino terminal $\mathrm{Tyr}^{42}$ of I $\mathrm{I} B-\alpha$ is sufficient to release NF- $\kappa \mathrm{B}$, although, unlike the classical phosphorylation of $\mathrm{Ser}^{32}$ and $\mathrm{Ser}^{36}$, this modification does not cause degradation of the inhibitor (37). The tyrosine phosphorylation of IKB- $\alpha$ was originally described in response to hypoxia-reperfusion injury, but it is possible that other stimuli, such as the ones related to atherogenesis, may also activate this kinase. Indeed, there is recent evidence that oxidative stress activates NF- $\kappa B$ through a mechanism that involves $\mathrm{Tyr}^{42}$ of IKB- $\alpha$ and is distinct from that stimulated by cytokines or growth factors (38). At present, the mechanisms by which the various proatherogenic signaling systems activate NF- $\mathrm{KB}$ remain an important challenge.

$\mathrm{NF}-\mathrm{KB}$ and the life and death of vascular cells The NF-KB system can be thought of as coordinating the activation of multiple inflammatory genes, but it may also induce genes whose products have survival and protective functions that are important in regulating the expansion and progression of atherosclerotic lesions. Activation of NF- $\mathrm{KB}$ is associated with resistance to apoptosis. One mechanism by which NF- $\mathrm{KB}$ inhibits cell death is to induce the expression of genes that promote resistance to apoptosis. NF- $\kappa B$ activation suppresses caspase activation by increasing expression of the inhibitors of apoptosis (IAP) proteins, c-IAP1, 
c-IAP2, and XIAP, as well as the TNF receptor-associated factors (TRAFs), TRAF1 and TRAF2 (39). NF- $\mathrm{KB}$ also suppresses mitochondrial cytochrome $c$ release through induction of a Bcl-2 family member. Thus, $\mathrm{NF}-\mathrm{KB}$ induces multiple factors to suppress cell death, blocking apoptosis at multiple steps along the cell death cascade. Although information on the expression of these inhibitors of apoptosis in the vessel wall is modest, they seem to have an emerging role in atherosclerosis. The coordinate induction of a set of genes by NF- $\kappa B$ that function cooperatively to suppress apoptosis may be important in preventing cell death in response to the proinflammatory signals associated with the onset of atherosclerosis.

In addition to the antiapoptotic genes, NF- $\mathrm{KB}$ may induce the expression of a set of protective genes in vascular cells. For example, protection against oxidative stress, which accompanies atherogenesis, may be accomplished by increasing expression of heme oxygenase-1 (HO-1) or manganese superoxide dismutase. HO- 1 is a heme-degradation enzyme, induced under conditions of oxidative stress, that produces the antioxidants biliverdin and bilirubin. The enzyme is induced by mildly oxidized LDL and diminishes the chemotactic response of monocytes exposed to oxidized LDL (40). In atherosclerotic lesions, expression of the enzyme is prominent in endothelial cells and foam cells of the thickened intima and can be detected in medial smooth muscle cells of advanced lesions (41). Induction of HO-1 by oxidized LDL may generate antioxidants and thereby protect against inflammation in the vessel wall.

The survival and protective genes may serve to limit the proinflammatory response and thereby regulate the response to injury. It is possible that when the vasculature is subjected to strong proatherogenic stimuli, the accumulation of reactive products results in the activation of the NF- $\mathrm{KB}$ system and the induction of a proinflammatory response. Low levels or episodic activation of NF- $\kappa B$ would be under tight control by constitutively expressed IKB. This would prevent inappropriate induction of a proinflammatory response by low levels of reactive oxygen or lipid species. Following a strong proatherogenic challenge, NF- $\mathrm{KB}$ activation would result in the induction of genes directing expression of adhesion molecules and chemokines. This response would result in the recruitment of leukocytes and the activation of an immune response to limit the injury. In parallel with the induction of the inhibitor, IKB, the proatherogenic challenge would be accompanied by the upregulation of both survival and protective genes. The genes would safeguard the vascular cells from apoptosis or injury caused by factors such as TNF- $\alpha$ or toxic reactive species that might be generated during the response. These products would supplement any constitutively expressed protective components and help restore the vascular cell to a quiescent state, despite the strong signals that elicited the response. However, in extreme proatherogenic conditions, even high levels of induced survival and protective genes may not be sufficient to counteract the intensity of the stimulation. Unbalanced activation would lead to cell injury and death, consistent with the increased levels of apoptosis seen in atherosclerotic lesions.

\section{Hemodynamics and the distribution} of atherosclerotic lesions

Atherosclerotic lesions form at distinct regions of the arterial tree, especially at or near branch points, bifurcations, and major curvatures. At bifurcations, disturbed laminar blood flow, with boundary layer separation, flow reversal, secondary flows, and shifting stagnation points can be encountered, and as a result, endothelial cells can be chronically exposed to shear forces with cyclical variation in direction (Figure 2). In this situation, the mean shear stress is relatively low. In contrast, straight segments of arteries, which are relatively protected from atherogenesis, experience uniform laminar flow and higher levels of mean shear stress. Regional differences in blood flow or hemodynamics are sensed by endothelium and can influence various biological parameters of these cells (reviewed in ref. 42).

Hemodynamic forces may predispose specific regions of the aorta to atherosclerotic lesion formation when the appropriate risk factors are present. For example, hemodynamic factors contribute to the accumulation of lipoprotein particles in the intima, and this phenomenon is accentuated by hyperlipidemia. However, it is not clear how the other systemic risk factors, such as hypertension, hyperglycemia, infectious agents, or other oxidative stresses including smoking, upregulate atherogenesis in specific regions of arteries, when the entire arterial tree is exposed. A potential explanation might hold that regional differences in NF- $\mathrm{KB}$ signaling preferentially transduce systemic stimuli, resulting in regional coordinate expression of proatherosclerotic genes. Such a model receives indirect support from the observation that VCAM-1, a NF- $\mathrm{KB}$-dependent gene product, is expressed in atherosclerosis-predisposed regions even in normocholesterolemic animals.

Recently we demonstrated dramatic regional differences in NF-KB signal transduction in mouse aortic endothelial cells using specific antibody staining and en face confocal microscopy (43). We have found that the expression of key NF- $\mathrm{KB} / \mathrm{I} \kappa \mathrm{B}$ components is markedly increased in a region of the ascending aorta and arch highly predisposed to atherosclerotic lesion formation relative to a protected site located just millimeters away. In control mice, NF- $\kappa \mathrm{B}$ is localized predominantly in the cytoplasm, and only a minority of endothelial cells show signs that NF- $\kappa B$ is active, suggesting that this signal transduction system is primed for activation in the lesion-predisposed region. LPS treatment or feeding $L D L R^{-/-}$mice an atherogenic diet 


\section{Figure 2}

$\mathrm{NF}-\mathrm{KB}$ and hemodynamics in regions predisposed and protected from atherosclerosis. Schematic of hemodynamics in the human carotid bifurcation. The straight segment of the artery proximal to the bifurcation is typical of regions resistant to atherogenesis (left). These regions are associated with a uniformly laminar blood flow profile that may induce the expression of various classes of atheroprotective genes. Examples of each class from the work of Akimoto et al. (46) and Gimbrone and coworkers (42) are shown (below left). It is not known whether this class of genes is coordinately regulated. In contrast, disturbed laminar flow, with boundary layer separation, flow reversal, secondary flows, and shifting stagnation points, is typical of regions predisposed to atherosclerosis (right). In these regions, the mean shear stress is relatively low and the NF- $\mathrm{KB}$ signal transduction pathway may be primed for activation. Systemic risk factors would therefore preferentially induce the expression of proatherogenic NF-KB-dependent genes at lesion-predisposed sites. Examples of each class are provided (below right).

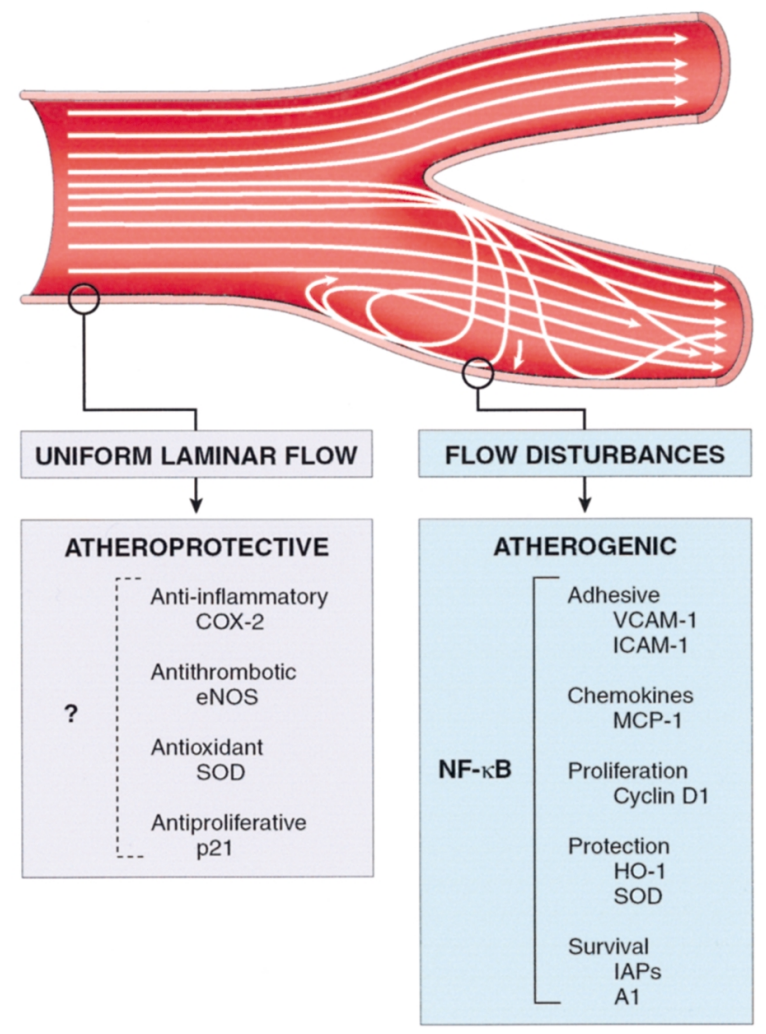

$\mathrm{NF}-\mathrm{KB}$ and entry into the cell cycle at sites of nonlaminar flow

NF- $\kappa B$ may be involved in the cell cycle entry that occurs at sites of atherosclerotic lesion formation. Studies of endothelial cell proliferation in vivo noted that although the fraction of proliferating endothelial cells in the arterial system is low, there are foci of proliferating cells associated with areas of disturbed flow, such as curvatures or bifurcations. In vitro models support these findings and demonstrate that disturbed flow causes substantial increases in cell-cycle entry (42). The spatial correlation between the locations of proliferating endothelium and the sites of NF- $\mathrm{KB}$ activation is consistent with recent data suggesting that this transcription factor is a positive mediator of cell growth. NF- $\mathrm{KB}$ activates cyclin D1 expression by direct binding of NF- $\kappa B$ to multiple sites in the cyclin $D 1$ promoter (24). Inhibition of the NF- $\kappa B$ system causes a reduction in cyclin D1-associated kinase activity, delayed phosphorylation of the retinoblastoma protein, and retarded G1/S transition, all consistent with the hypothesis that NF- $\mathrm{KB}$ contributes to cell-cycle progression at sites of atherosclerotic lesion formation.

The link between the cell cycle and the spatial hemodynamics of focal atherogenesis was strengthened by recent studies demonstrating that laminar shear stress induces the cyclin-dependent kinase inhibitor p21 and suppresses the G0/G1 to $S$ phase transition (46). Thus, 
at sites in the aorta subjected to laminar shear stress, increased levels of p21 may block endothelial proliferation, whereas, in the disturbed-flow regions of the aorta that are typically associated with atherosclerotic lesion formation, suppression of p21 would favor endothelial proliferation (Figure 2). This model suggests that at sites of lesion formation, NF- $\kappa \mathrm{B}$ may induce genes like cyclin D1 that stimulate cell-cycle entry, and in parallel, levels of the p21 may fall, activating cyclin-dependent kinases. Collectively, these events would stimulate endothelial cells subjected to flow disturbances to enter into the cell cycle.

\section{$\mathrm{NF}-\mathrm{KB}$ and the atheroprotective gene hypothesis}

Atheroprotective genes have been proposed to suppress the pathogenesis of atherosclerosis. The hypothesis states that the coordinated induction of a subset of endothelial genes (e.g., anti-inflammatory, antithrombotic, and antioxidant) in response to laminar shear stress could exert a net vasoprotective effect. Disturbed or turbulent flow, a nonlaminar fluid mechanical stimulus, may fail to induce these genes, predisposing the endothelium to dysfunction and the development of atherosclerotic lesions (reviewed in ref. 42).

The regulation of the NF- $\mathrm{KB}$ system may be an important aspect of the atheroprotective concept. At sites of disturbed blood flow, NF- $\mathrm{\kappa B}$ could coordinately induce a select group of proatherogenic or "atheropermissive" genes that offset the vasoprotective effect of the atheroprotective genes (Figure 2). Areas of the aorta that have a low probability of lesion formation may be protected from NF- $\kappa B$ activation. Indeed, levels of NF- $\mathrm{KB}$ components and the extent of NF- $\mathrm{\kappa B}$ activation are low in regions of the mouse aorta with a low probability of lesion formation. Moreover, in vitro studies have shown that laminar shear stress stimulation of cultured endothelial cells can diminish inflammatory cytokine-induced expression of VCAM-1, although the mechanism of this suppression is unclear. Interactions between the products of the atheroprotective genes and the NF- $\mathrm{KB}$ signaling system may also be important. For example, one of the candidate atheroprotective genes preferentially induced by laminar sheer stress encodes the endothelial isoform of $\mathrm{NO}$ synthase (eNOS) (42). This enzyme may generate NO which, in turn, can modulate vasomotor tone, inhibit platelet aggregation, and inhibit smooth muscle migration. Additionally, $\mathrm{NO}$ diminishes inflammatory gene expression by inducing and stabilizing the NF- $\mathrm{\kappa B}$ inhibitor I $\kappa B-\alpha$ (47). This may contribute to the suppression of the NF- $\mathrm{KB}$ signaling system in the protected regions of the aorta. Another relevant interaction might be with the atheroprotective antioxidants. Hypertension has proinflammatory actions, increasing the formation of hydrogen peroxide and free radicals such as superoxide anion and hydroxyl radicals in plasma. At sites of laminar sheer stress, levels of the athero- protective antioxidants would be high, protecting the vessel wall. In contrast, at sites of disturbed flow where the levels of atheroprotective antioxidants are low, these reactive substances may reduce the formation of NO by endothelium and activate NF- $\kappa$ B. Collectively, these changes would lead to atherogenic changes and altered vascular tone at specific sites in the vessel wall.

\section{Is NF- $\mathrm{KB}$ a key mediator in atherogenesis?}

A remarkable series of correlations between the onset of atherogenesis and activation of NF- $\kappa B$ suggest that the $\mathrm{NF}-\mathrm{KB} / \mathrm{I} \kappa \mathrm{B}$ regulatory system plays a key role in this disease process. These correlations include the presence of activated NF- $\mathrm{KB}$ in atherosclerotic lesions but not in normal vessels and the induction of NF- $\mathrm{KB}$-dependent genes during atherogenesis. Additionally, the atherosclerotic lesion develops in response to diverse stimuli that have in common the ability to create oxidant stress and activate the NF-KB system; conversely, agents that inhibit lesion formation often act as antioxidants and stabilize the system.

Although these correlations are provocative, there is no direct evidence demonstrating that NF- $\mathrm{KB}$ activation is necessary for atherosclerosis. Standard approaches to establishing a causal link between NF-KB activation and atherogenesis would employ genetic gain or loss of function mutants. Unfortunately, mice with targeted mutations in the $p 65$ and $I \kappa B \alpha$ genes die during development, making it difficult to test the role of these proteins in the formation of lesions in mouse models of atherogenesis. To circumvent these issues, cell type-specific conditional mutations could be generated using Cre-loxP technology. In this approach, vascular cell-specific promoters would direct the expression of Cre recombinase, which would, in turn, direct the selective inactivation of specific genes in the NF- $\mathrm{KB}$ signaling system only in vascular cells. Progress is being made in defining the regulatory regions necessary for these studies and in generating lines of mice with target genes, such as $p 65$ and $I K K-\gamma$ suitably modified with loxP sites. In gain of function approaches, the identification of individual components of the NF- $\kappa B$ signaling cascade provides molecular targets for cell type-specific overexpression studies. Collectively, these approaches should allow a rigorous test of the link between the activation of this transcription factor pathway and the cellular events in the pathogenesis of atherosclerosis.

\section{Acknowledgments}

The authors would like to acknowledge J. Boucher for help with the manuscript and J. Perkins for preparation of the illustrations. In revising this manuscript for publication as part of the JCI Perspective series on NF- $\kappa B$, we have had to substantially reduce the number of citations. We have tried to highlight recent contributions, and apologize to the many friends in the field whose work we could not directly acknowledge due to space 
limitations. A more fully referenced version of the review is available upon request from $\mathrm{T}$. Collins. This work was supported by the NIH (grants P50 HL56985, PO1 HL36028, and RO1 HL45462) and by the Heart and Stroke Foundation of Ontario (grant T-3588) and the Canadian Institutes of Health Research (grant 14151). M.I. Cybulsky holds an Established Investigatorship from the American Heart Association.

1. Ross, R. 1999. Atherosclerosis: an inflammatory disease. N. Engl. J. Med. 340:115-126.

2. Karin, M. 1999. The beginning of the end: IkappaB kinase (IKK) and NFkappaB activation. J. Biol. Chem. 274:27339-27342.

3. Brand, K., et al. 1996. Activated transcription factor nuclear factor-kappa $\mathrm{B}$ is present in the atherosclerotic lesion. J. Clin. Invest. 97:1715-1722.

4. Wilson, S.H., et al. 2000. Activated nuclear factor-kappaB is present in the coronary vasculature in experimental hypercholesterolemia. Atherosclerosis. 148:23-30.

5. Iivama, K., et al. 1999. Patterns of vascular cell adhesion molecule-1 and intercellular adhesion molecule- 1 expression in rabbit and mouse atherosclerotic lesions and at sites predisposed to lesion formation. Circ. Res. 85:199-207.

6. Cybulsky, M.I., and Gimbrone, M.A., Jr. 1991. Endothelial expression of a mononuclear leukocyte adhesion molecule during atherogenesis. Science. 251:788-791.

7. Boring, L., Gosling, J., Cleary, M., and Charo, I.F. 1998. Decreased lesion formation in CCR2-/- mice reveals a role for chemokines in the initiation of atherosclerosis. Nature. 394:894-897.

8. Gu, L., et al. 1998. Absence of monocyte chemoattractant protein-1 reduces atherosclerosis in low density lipoprotein receptor-deficient mice. Mol. Cell. 2:275-281.

9. Li, N., and Karin, M. 1999. Is NF-kappaB the sensor of oxidative stress? FASEB J. 13:1137-1143.

10. Brand, K., et al. 1997. Dysregulation of monocytic nuclear factor-kappa B by oxidized low-density lipoprotein. Arterioscler. Thromb. Vasc. Biol. 17:1901-1909.

11. Khan, B.V., Parthasarathy, S.S., Alexander, R.W., and Medford, R.M. 1995. Modified low-density lipoprotein and its constituents augment cytokineactivated vascular cell adhesion molecule- 1 gene expression in human vascular endothelial cells. J. Clin. Invest. 95:1262-1270.

12. Palmetshofer, A., Robson, S.C., and Nehls, V. 1999. Lysophosphatidic acid activates nuclear factor kappa B and induces proinflammatory gene expression in endothelial cells. Thromb. Haemost. 82:1532-1537.

13. Calara, F., et al. 1998. An animal model to study local oxidation of LDL and its biological effects in the arterial wall. Arterioscler. Thromb. Vasc. Biol. 18:884-893.

14. Dichtl, W., et al. 1999. Very low-density lipoprotein activates nuclear factor-kappaB in endothelial cells. Circ. Res. 84:1085-1094.

15. Tontonoz, P., Nagy, L., Alvarez, J.G., Thomazy, V.A., and Evans, R.M. 1998. PPARgamma promotes monocyte/macrophage differentiation and uptake of oxidized LDL. Cell. 93:241-252.

16. Li, A.C., et al. 2000. Peroxisome proliferator-activated receptor $\gamma$ ligands inhibit development of atherosclerosis in LDL receptor-deficient mice.J. Clin. Invest. 106:523-531.

17. Pueyo, M.E., et al. 2000 . Angiotensin II stimulates endothelial vascular cell adhesion molecule-1 via nuclear factor-kappaB activation induced by intracellular oxidative stress. Arterioscler. Thromb. Vasc. Biol. 20:645-651.

18. Han, Y., Runge, M.S., and Brasier, A.R. 1999. Angiotensin II induces interleukin-6 transcription in vascular smooth muscle cells through pleiotropic activation of nuclear factor-kappa B transcription factors. Circ. Res. 84:695-703

19. Tummala, P.E., et al. 1999. Angiotensin II induces vascular cell adhesion molecule- 1 expression in rat vasculature: a potential link between the renin-angiotensin system and atherosclerosis. Circulation. 100:1223-1229.

20. Schmidt, A.M., Yan, S.D., Wautier, J.L., and Stern, D. 1999. Activation of receptor for advanced glycation end products: a mechanism for chronic vascular dysfunction in diabetic vasculopathy and atherosclerosis. Circ. Res. 84:489-497.

21. Morigi, M., et al. 1998. Leukocyte-endothelial interaction is augmented by

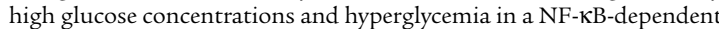

fashion. J. Clin. Invest. 101:1905-1915.

22. Welch, G.N., and Loscalzo, J. 1998. Homocysteine and atherothrombosis. N. Engl. J. Med. 338:1042-1050.

23. Outinen, P.A., et al. 1999. Homocysteine-induced endoplasmic reticulum stress and growth arrest leads to specific changes in gene expression in human vascular endothelial cells. Blood. 94:959-967.

24. Guttridge, D.C., Albanese, C., Reuther,J.Y., Pestell, R.G., and Baldwin, A.S., Jr. 1999. NF-kappaB controls cell growth and differentiation through transcriptional regulation of cyclin D1. Mol. Cell. Biol. 19:5785-5799.

25. Pruefer, D., Scalia, R., and Lefer, A.M. 1999. Homocysteine provokes leukocyte-endothelium interaction by downregulation of nitric oxide. Gen Pharmacol. 33:487-498.

26. Libby, P., Egan, D., and Skarlatos, S. 1997. Roles of infectious agents in atherosclerosis and restenosis: an assessment of the evidence and need for future research. Circulation. 96:4095-4103.

27. Speir, E., Shibutani, T., Yu, Z.X., Ferrans, V., and Epstein, S.E. 1996. Role of reactive oxygen intermediates in cytomegalovirus gene expression and in the response of human smooth muscle cells to viral infection. Circ. Res. 79:1143-1152.

28. Hiscott, J., Kwon, H., and Génin, P. 2001. Hostile takeovers: viral appropriation of the NF-KB pathway. J. Clin. Invest. 107:143-151.

29. Knight, D.A., Waldman, W.J., and Sedmak, D.D. 1999. Cytomegalovirusmediated modulation of adhesion molecule expression by human arterial and microvascular endothelial cells. Transplantation. 68:1814-1818.

30. Nicholson, A.C., and Hajjar, D.P. 1998. Herpesvirus in atherosclerosis and thrombosis: etiologic agents or ubiquitous bystanders? Arterioscler. Thromb. Vasc. Biol. 18:339-348.

31. Kol, A., Sukhova, G.K., Lichtman, A.H., and Libby, P. 1998. Chlamydial heat shock protein 60 localizes in human atheroma and regulates macrophage tumor necrosis factor-alpha and matrix metalloproteinase expression. Circulation. 98:300-307.

32. De Caterina, R., et al. 1999. The inhibition of endothelial activation by unsaturated fatty acids. Lipids. 34:S191-S194.

33. Diaz, M.N., Frei, B., Vita, J.A., and Keaney, J.F., Jr. 1997. Antioxidants and atherosclerotic heart disease. N. Engl. J. Med. 337:408-416.

34. Erl, W., Weber, C., Wardermann, C., and Weber, P.C. 1997. alpha-Tocopheryl succinate inhibits moncytic cell adhesion to endothelial cells by suppressing NF-kappa B mobilization. Am. J. Physiol. 273:H634-H640.

35. Nunes, G.L., et al. 1997. Vitamins $\mathrm{C}$ and $\mathrm{E}$ inhibit $\mathrm{O}_{2}$-production in the pig coronary artery. Circulation. 96:3593-3601.

36. Peters, R., Liao, S.M., and Maniatis, T. 2000. IKKe is part of a novel PMAinducible IкB kinase complex. Mol. Cell. 5:513-522.

37. Imbert, V., et al. 1996. Tyrosine phosphorylation of I kappa B-alpha activates NF-kappa B without proteolytic degradation of I kappa B-alpha. Cell. 86:787-798.

38. Schoonbroodt, S., et al. 2000. Crucial role of the amino-terminal tyrosine residue 42 and the carboxyl-terminal PEST domain of IkappaBalpha in NF-kappa B activation by an oxidative stress. J. Immunol. 164:4292-4300.

39. Wang, C.Y., Mayo, M.W., Korneluk, R.G., Goeddel, D.V., and Baldwin, A.S., Jr. 1998. NF-kappaB antiapoptosis: induction of TRAF1 and TRAF2 and c-IAP1 and C-IAP2 to suppress caspase-8 activation. Science. 281:1680-1683

40. Ishikawa, K., Navab, M., Leitinger, N., Fogelman, A.M., and Lusis, A.J. 1997. Induction of heme oxygenase- 1 inhibits the monocyte transmigration induced by mildly oxidized LDL. Clin. Invest. 100:1209-1216.

41. Wang, L.J., Lee, T.S., Lee, F.Y., Pai, R.C., and Chau, L.Y. 1998. Expression of geme oxygenase- 1 in atherosclerotic lesions. Am. J. Pathol. 152:711-720.

42. Gimbrone, M.A., Jr. 1999. Vascular endothelium, hemodynamic forces, and atherogenesis. Am. J. Pathol. 155:1-5.

43. Hajra, L., et al. 2000. The NF- $\mathrm{KB}$ signal transduction pathway in aortic endothelial cells is primed for activation in regions predisposed to atherosclerotic lesion formation. Proc. Natl. Acad. Sci. USA. 97:9052-9057.

44. Mohan, S., Mohan, N., and Sprague, E.A. 1997. Differential activation of NF-kappa B in human aortic endothelial cells conditioned to specific flow environments. Am. J. Physiol. 273:C572-C578.

45. Bhullar, I.S., et al. 1998. Fluid shear stress activation of IkappaB kinase is integrin-dependent. J. Biol. Chem. 273:30544-30549.

46. Akimoto, S., Mitsumata, M., Sasaguri, T., and Yoshida, Y. 2000. Laminar shear stress inhibits vascular endothelial cell proliferation by inducing cyclin-dependent kinase inhibitor p21. Circ. Res. 86:185-190.

47. Peng, H.B., Libby, P., and Liao, J.K. 1995. Induction and stabilization of I kappa B alpha by nitric oxide mediates inhibition of NF-kappa B. J. Biol. Chem. 270:14214-14219. 